\title{
Packing tree factors in random and pseudo-random graphs
}

\author{
Deepak Bal \\ Department of Mathematics \\ Ryerson University \\ Toronto, ON, M5B 2K3, Canada \\ deepak.c.bal@ryerson.ca \\ Michael Krivelevich ${ }^{\dagger}$ \\ School of Mathematical Sciences \\ Tel Aviv University \\ Tel Aviv, 69978, Israel \\ krivelev@post.tau.ac.il
}

\author{
Alan Frieze* \\ Department of Mathematical Sciences \\ Carnegie Mellon University \\ Pittsburgh, PA 15213, U.S.A. \\ alan@random.math. cmu.edu \\ Po-Shen Loh ${ }^{\ddagger}$ \\ Department of Mathematical Sciences \\ Carnegie Mellon University \\ Pittsburgh, PA 15213, U.S.A. \\ ploh@cmu.edu
}

Submitted: Apr 9, 2013; Accepted: Apr 2, 2014; Published: Apr 16, 2014

Mathematics Subject Classifications: 05C70; 05C05; 05C80

\begin{abstract}
For a fixed graph $H$ with $t$ vertices, an $H$-factor of a graph $G$ with $n$ vertices, where $t$ divides $n$, is a collection of vertex disjoint (not necessarily induced) copies of $H$ in $G$ covering all vertices of $G$. We prove that for a fixed tree $T$ on $t$ vertices and $\epsilon>0$, the random graph $G_{n, p}$, with $n$ a multiple of $t$, with high probability contains a family of edge-disjoint $T$-factors covering all but an $\epsilon$-fraction of its edges, as long as $\epsilon^{4} n p \gg \log ^{2} n$. Assuming stronger divisibility conditions, the edge probability can be taken down to $p>\frac{C \log n}{n}$. A similar packing result is proved also for pseudorandom graphs, defined in terms of their degrees and co-degrees.
\end{abstract}

Keywords: Tree factors; Packing; Random graphs; Pseudo-random graphs

\footnotetext{
* Research supported in part by NSF Grant CCF2013110.

${ }^{\dagger}$ Research supported in part by a USA-Israel BSF grant and by a grant from the Israel Science Foundation.

${ }^{\ddagger}$ Research supported by NSF grant DMS-1201380, an NSA Young Investigators Grant, and by a USA-Israel BSF grant.
} 


\section{Introduction}

Let $H$ be a graph with $t$ vertices and let $n$ be divisible by $t$. We say that a graph $G=(V, E)$ with $n$ vertices has an $H$-factor if there exist vertex disjoint subgraphs (not necessarily induced) of $G, H_{1}, \ldots, H_{n / t}$, which are all isomorphic to $H$. Note that the vertex disjointness implies that the vertex set of the $H$-factor, $H_{1} \cup \cdots \cup H_{n / t}$, is equal to $V$. $H$-factors have been an important object in the study of random graphs. Indeed, the most basic instance, a $K_{2}$-factor, corresponds to a perfect matching. Erdős and Rényi [3] proved in 1966 that if $p=\frac{\log n+\omega}{n}$, with $\omega \rightarrow \infty$ and $n$ even, then the Erdős-Rényi-Gilbert random graph, $G_{n, p}$ has a perfect matching $\mathbf{w h p}^{1}$. In 1981, Shamir and Upfal [17] proved a general result which implies that if $p=\frac{\log n+(r-1) \log \log n+\omega}{n}$, with $\omega \rightarrow \infty$ arbitrarily slowly, and $n$ even, then $G_{n, p}$ contains $r$ edge-disjoint perfect matchings whp. In this range of $p$, the minimum degree of $G_{n, p}$ is $r \mathbf{w h p}$, so the result is optimal. Luczak and Ruciński [15], as a corollary of a more technical result, proved that for any tree $T$, if $p=\frac{\log n+\omega}{n}$ with $\omega \rightarrow \infty$, and $n$ is divisible by $|T|$, then $G_{n, p}$ has a $T$-factor whp. As an analogue to the theorem of Shamir and Upfal, Kurkowiak [14] proved that if $p=\frac{\log n+(r-1) \log \log n+\omega}{n}$ with $\omega \rightarrow \infty$, then $G_{n, p}$ contains $r$ edge-disjoint $T$-factors whp.

The study of optimal and near-optimal packings of spanning objects in graphs and hypergraphs is an area of much active research. Recently, the case of Hamilton cycles (simple spanning cycles) has been the subject of many papers. When $p=\frac{\log n+(2 r-1) \log \log n+\omega}{n}$ where $\omega \rightarrow \infty$, Bollobás and Frieze [2] proved in 1985 that $G_{n, p}$ contains $r$ edge-disjoint Hamilton cycles whp. In [4], Frieze and Krivelevich conjectured that for any $0<p=$ $p(n) \leqslant 1, G_{n, p}$ contains $\lfloor\delta / 2\rfloor$ edge-disjoint Hamilton cycles whp, where $\delta$ represents the minimum degree. The conjecture was solved in the series of papers [5],[11],[12] and [13]. In intermediate papers such as [4] and [10], the notion of approximate or almost optimal packings was studied. The results in these papers state that for certain ranges for $p$, all but a vanishing fraction of the edges of $G_{n, p}$ can be covered with edge-disjoint Hamilton cycles.

In this work, we investigate when all but a vanishing fraction of the edges of random and pseudo-random graphs be covered with edge-disjoint $T$-factors, for a fixed tree $T$. We begin by introducing the notion of pseudo-randomness which we will use in this paper.

Definition 1.1. Let $G=(V, E)$ be a graph with $n$ vertices. We say $G$ is $(\boldsymbol{\epsilon}, \boldsymbol{p})$-regular if the following 2 conditions hold:

- $d(v) \geqslant(1-\epsilon) n p$ for every vertex $v$.

- $d(u, v) \leqslant(1+\epsilon) n p^{2}$ for every pair of distinct vertices $u$ and $v$.

Here, $d(v)$ denotes the degree of vertex $v$, and $d(u, v)$ denotes the co-degree of $u$ and $v$, i.e., the number of neighbors common to both $u$ and $v$. We will also write $d_{S}(v)$ and $d_{S}(v, w)$ to refer to the degree and the co-degree into a set $S$ of vertices. Our pseudo-randomness conditions are localized, and this is in part necessary because we are packing spanning structures. Nevertheless, these conditions are satisfied whp by $G_{n, p}$, for appropriately

\footnotetext{
${ }^{1}$ An event $\mathcal{E}_{n}$ occurs with high probability, or whp, if $\lim _{n \rightarrow \infty} \mathbb{P}\left[\mathcal{E}_{n}\right]=1$.
} 
chosen $\epsilon$. Indeed, by the standard Chernoff bound, stated as Theorem 2.1 in the next section:

$$
\begin{aligned}
& \mathbb{P}\left[G_{n, p} \text { is not }(\epsilon, p) \text {-regular }\right] \\
& <n \mathbb{P}[\operatorname{Bin}[n-1, p]<(1-\epsilon) n p]+n^{2} \mathbb{P}\left[\operatorname{Bin}\left[n-2, p^{2}\right]>(1+\epsilon) n p^{2}\right] \\
& =o(1),
\end{aligned}
$$

as long as $\epsilon^{2} n p^{2} \gg \log n$. (In this paper, we will write $A_{n} \gg B_{n}$ when $A_{n} / B_{n} \rightarrow \infty$ with $n$.) Our theorem for $(\epsilon, p)$-regular graphs is then as follows.

Theorem 1. Let $T$ be a fixed tree with $t$ vertices, and let $G$ be an $(\epsilon, p)$-regular graph on $n$ vertices, with $n$ a multiple of $t$. If $\epsilon, n$ and $p$ satisfy $\epsilon^{6} n p^{4} \gg \log ^{3} n$ then for $n$ sufficiently large, $G$ contains a collection of edge-disjoint $T$-factors covering all but $2 \epsilon^{1 / 3}$-fraction of its edges.

For random graphs, we have two results. Let $\mathcal{P}(\epsilon)$ be the graph property that all but an $O(\epsilon)$-fraction of the edges of a graph may be $n p$ is above a certain power of $n$, random graphs $G_{n, p}$ can be almost packed with tree-factors. That initial range is not optimal, and resembles the barrier which was hit during the investigation of Hamilton cycle packing in random structures (see, e.g., [1], [4], and [7]). Using additional properties of $G_{n, p}$, we are able to push the result to smaller $p$.

Theorem 2. Let $T$ be a fixed tree with $t$ vertices. If $\epsilon, n$, and $p$ satisfy $\epsilon^{4} n p \gg \log ^{2} n$, then $G_{n, p}$, with $n$ a multiple of $t$, satisfies $\mathcal{P}(\epsilon)$ whp.

This range of $p\left(\gg \frac{\log ^{2} n}{n}\right)$ is still probably not optimal, and in the context of Hamilton cycle packing, it took further developments to remove the last logarithmic factors. For tree-factor packing, however, it turns out that we can circumvent this obstacle. In the following theorem, we improve the range of $p$ to asymptotically best possible, subject to an additional divisibility condition on $n$, which we suspect to be an artifact of our proof technique.

Theorem 3. Given any t-vertex tree $T$ and any positive real $\epsilon$, there exists an integer $\tau_{0}$ such that for any $\tau \geqslant \tau_{0}$ satisfying $t \mid \tau$, there is a real constant $C$ such that for $p>\frac{C \log n}{n}$, the random graph $G_{n, p}$ satisfies $\mathcal{P}(\epsilon)$ whp for $\tau \mid n$.

The complexity of the above result stems from the fact that it is stated in greater generality. Indeed, note that if one applies it with the particular choice $\tau=\tau_{0}$, then the conclusion is that there is a real $C_{0}$ such that $G_{n, p}$ satisfies $\mathcal{P}(\epsilon)$ whp for $\tau_{0} \mid n$, when $p>\frac{C_{0} \log n}{n}$. This is within a factor $\left(C_{0}\right)$ of the best possible result, and the divisibility condition is also off by a factor (ideally, it would only require $t \mid n$ ). Although it may be more challenging to eliminate $C_{0}$, we conjecture that perhaps it may not be as difficult to relax the divisibility condition.

Conjecture 1. Given any t-vertex tree $T$ and any positive real $\epsilon$, there exists a real constant $C$ such that for $p>\frac{C \log n}{n}$, the random graph $G_{n, p}$ satisfies $\mathcal{P}(\epsilon)$ whp for $t \mid n$. 
Throughout our exposition, we will implicitly assume that $\epsilon$ is sufficiently small and $n$ is sufficiently large. The following (standard) asymptotic notation will be utilized extensively. For two functions $f(n)$ and $g(n)$, we write $f(n)=o(g(n))$ if $\lim _{n \rightarrow \infty} f(n) / g(n)=0$, and $f(n)=O(g(n))$ if there exists a constant $M$ such that $|f(n)| \leqslant M|g(n)|$ for all sufficiently large $n$. We also write $f(n)=\Theta(g(n))$ if both $f(n)=O(g(n))$ and $g(n)=O(f(n))$ are satisfied. We also write $A=(1 \pm \epsilon) B$ to mean $(1-\epsilon) B \leqslant A \leqslant(1+\epsilon) B$. All logarithms will be in base $e \approx 2.718$.

\section{Concentration inequalities}

For the reader's convenience, we record in this section the two large-deviation bounds which we will use in this paper. We will appeal to the following version of the Chernoff bound, which can be found, for example, as Corollary 2.3 in the book by Janson, Łuczak, and Ruciński [9].

Theorem 2.1. Let $X$ be a binomial random variable with mean $\mu$, and let $0<\epsilon<1$. Then

$$
\mathbb{P}[|X-\mu|>\epsilon \mu] \leqslant 2 e^{-\epsilon^{2} \mu / 3} .
$$

The previous result establishes concentration of a random variable defined over a product space. In this paper, we will also encounter a particular non-product space. For that, we use the following concentration bound which applies in the setting where the probability space is the uniform distribution over permutations of $n$ elements. For a proof, we refer the reader to [8] or [16].

Theorem 2.2. Let $X$ be a random variable determined by a uniformly random permutation on $n$ elements, and let $C$ be a real number. Suppose that whenever $\sigma, \sigma^{\prime} \in S_{n}$ differ by a single transposition, $\left|X(\sigma)-X\left(\sigma^{\prime}\right)\right| \leqslant C$. Then,

$$
\mathbb{P}[|X-\mathbb{E}[X]| \geqslant t] \leqslant 2 \exp \left\{-\frac{2 t^{2}}{C^{2} n}\right\} .
$$

\section{Proof of Theorem 1}

Let $G=(V, E)$ be a graph on vertex set $V=[n]$, and suppose $t$ divides $n$ with $\nu=n / t$. Let $T=\left(V_{T}, E_{T}\right)$ be a fixed tree on vertex set $V_{T}=[t]$. Let $\sigma$ be a permutation of $[n]$. Let $G_{\sigma}=\left(V_{\sigma}, E_{\sigma}\right)$ be the $t$-partite subgraph of $G$ with vertex set

$$
V_{\sigma}=V_{\sigma, 1} \dot{\cup} V_{\sigma, 2} \dot{U} \cdots \dot{U} V_{\sigma, t}
$$

where $V_{\sigma, i}=\{\sigma((i-1) \nu+1), \ldots, \sigma(i \nu)\}$ for $i=1, \ldots, t$. The edge set is defined as

$$
E_{\sigma}=\left\{(u, v) \in E: \exists i, j \in[t] \text {, with }(i, j) \in E_{T} \text { and } u \in V_{\sigma, i}, v \in V_{\sigma, j}\right\} .
$$

In words, we use $\sigma$ to define a partition of $V$ into $t$ parts, corresponding to the vertices of $T$, and we keep the edges of $G$ which connect two parts corresponding to the endpoints 
of an edge of $T$. We also separately define $G_{\sigma}^{\prime}$ as the subgraph of $G$ where we keep edges between all pairs $\left(V_{\sigma, i}, V_{\sigma, j}\right)$, but still delete the edges within the $V_{\sigma, i}$.

The resulting $G_{\sigma}$ looks like a "blown-up" version of $T$. We call a pair $\left(V_{\sigma, i}, V_{\sigma, j}\right)$ a super-edge if $(i, j) \in E_{T}$, and we say that it is an $(\epsilon, p)$-regular pair if

- for all $v \in V_{\sigma, i}$ and $w \in V_{\sigma, j}, d_{V_{\sigma, j}}(v), d_{V_{\sigma, i}}(w) \geqslant(1-\epsilon) \nu p$, and

- for all $v, w \in V_{\sigma, i}$ and $v^{\prime}, w^{\prime} \in V_{\sigma, j}, d_{V_{\sigma, j}}(v, w), d_{V_{\sigma, i}}\left(v^{\prime}, w^{\prime}\right) \leqslant(1+\epsilon) \nu p^{2}$.

We say that $G_{\sigma}$ is an $(\epsilon, p)$-regular blow up of $T$ if every super-edge is an $(\epsilon, p)$-regular pair. Conveniently, if we take an $(\epsilon, p)$-regular graph $G$, and uniformly sample a random permutation $\sigma$ of $[n]$, then we typically preserve the regularity across super-edges in $G_{\sigma}$. Formally, we have:

Lemma 3.1. Let $G$ be an $(\epsilon, p)$-regular graph on $n$ vertices with $n$ divisible by $t$ and $\nu=n / t$. Suppose that $\epsilon^{2} n p^{4} \gg \log n$. Let $\sigma$ be a uniformly random permutation on $n$ elements, and define $G_{\sigma}$ as above. Then with probability $1-o\left(n^{-1}\right), G_{\sigma}$ is a $(2 \epsilon, p)$-regular blow up of $T$.

Proof. We will show that all pairs $\left(V_{\sigma, i}, V_{\sigma, j}\right)$ in $G_{\sigma}^{\prime}$ are $(2 \epsilon, p)$-regular pairs, which obviously implies the result for $G_{\sigma}$ since $G_{\sigma}$ and $G_{\sigma}^{\prime}$ agree on super-edges. We first show that all degrees are typically correct. Let $v$ be an arbitrary vertex and expose only the position of $v$ under $\sigma$. Suppose this reveals that $v \in V_{\sigma, i}$. Consider the pair $\left(V_{\sigma, i}, V_{\sigma, j}\right)$ in $G_{\sigma}^{\prime}$ for any $j \neq i$. Let $N_{v}:=d_{V_{\sigma, j}}(v)$ and note that this is a random variable whose randomness comes from the permutation $\sigma$. Conditioned on the position of $v, \sigma$ is a uniform random permutation on the $n-1$ remaining vertices and so every other vertex has probability $\frac{n / t}{n-1}=\frac{\nu}{n-1}$ of being in $V_{\sigma, j}$. We also know that $d_{G}(v) \geqslant(1-\epsilon) n p$ by $(\epsilon, p)$-regularity, so $\mathbb{E}\left[N_{v}\right] \geqslant(1-1.5 \epsilon) \nu p$.

For concentration we apply Theorem 2.2 to $N_{v}$. Note that transposing two elements of $\sigma$ can only change $N_{v}$ by at most 1 . So the probability that $N_{v}$ differs from its mean by more than $.5 \epsilon \nu p$ is bounded above by

$$
2 \exp \left\{-\frac{2(.5 \epsilon \nu p)^{2}}{n-1}\right\}=o\left(n^{-K}\right)
$$

for any positive constant $K$ as long as $\epsilon^{2} n p^{2} \gg \log n$. So taking a union bound over all vertices and choices of $j$ for $V_{\sigma, j}$, we have the degree conclusion of the lemma.

For co-degrees, we proceed similarly. Let $v$ and $w$ be arbitrary vertices and expose the positions of these two vertices under $\sigma$. Suppose this reveals that $v \in V_{\sigma, i}$ and $w \in V_{\sigma, j}$. Let $k \in[t]$ be distinct from $i$ and $j$. Note that we are really only concerned with the case when $i=j$ and $\left(V_{\sigma, i}, V_{\sigma, k}\right)$ is a super-edge, but this does not matter much. Let $N_{v, w}$ be the co-degree of $v$ and $w$ into $V_{\sigma, k}$ in $G_{\sigma}^{\prime}$. Conditioned on the positions of $v$ and $w, \sigma$ is a uniform random permutation on the $n-2$ remaining vertices, so every other vertex has probability $\frac{\nu}{n-2}$ of being in $V_{\sigma, k}$. Also $d_{G}(v, w) \leqslant(1+\epsilon) n p^{2}$ by $(\epsilon, p)$-regularity, so $\mathbb{E}\left[N_{v, w}\right] \leqslant(1+1.5 \epsilon) \nu p^{2}$. 
Applying Theorem 2.2, we have $N_{v, w} \leqslant(1+2 \epsilon) \nu p^{2}$ with probability at least $1-o\left(n^{-K}\right)$ for any positive constant $K$ as long as $\epsilon^{2} n p^{4} \gg \log n$. Taking a union bound over pairs of vertices and choices of $k$ for $V_{\sigma, k}$, we have the co-degree conclusion of the lemma.

We now define a procedure for generating edge-disjoint subgraphs of an $(\epsilon, p)$-regular graph $G$, each of which looks something like a $G_{\sigma}$.

Procedure 1. This procedure takes as input a graph $G=(V, E)$ on $n$ vertices with $n$ divisible by $t$. Let

$$
r=\frac{30}{\epsilon^{2}} \frac{t^{2}}{t-1} \log n
$$

and perform the following steps.

$\boldsymbol{P} 1$ Generate $r$ independent uniformly random permutations $\sigma_{1}, \ldots, \sigma_{r}$ of $[n]$. Construct $G_{1}=G_{\sigma_{1}}, \ldots, G_{r}=G_{\sigma_{r}}$ as described above.

P2 For each edge $e \in E$, let $L_{e}=\left\{i: e \in G_{i}\right\}$. If $L_{e} \neq \emptyset$, select a uniformly random element $i$ of $L_{e}$, and label $e$ with $i$.

P3 Let $\widehat{G}_{i}=\left(\widehat{V}_{i}, \widehat{E}_{i}\right)$ be the subgraph of $G_{i}$ consisting of all edges which received label $i$.

Note that the $\widehat{G}_{i}$ 's are now edge-disjoint by construction. Our goal will be to prove that the $\widehat{G}_{i}$ 's have regularity properties similar to those of the $G_{i}$ 's, but with larger $\epsilon$ and smaller $p$.

Lemma 3.2. Run Procedure 1 on an $(\epsilon, p)$-regular graph $G$ with $n$ vertices, where $n$ is divisible by $t$. Then with probability $1-o\left(n^{-1}\right)$, each edge $e \in G$ appears in $(1 \pm \epsilon) \kappa$ of the $G_{i}$ 's, where

$$
\kappa=\frac{60}{\epsilon^{2}} \log n=\frac{2(t-1)}{t^{2}} r .
$$

Proof. We first compute the probability $q$ that an edge $e=(v, w)$ appears in $G_{\sigma}$, when $\sigma$ is a uniformly random permutation. Then if we let $X_{e}$ be the random variable counting the number of $G_{i}$ 's which contain $e$, by the independence of the permutations $\sigma_{1}, \ldots, \sigma_{r}$, we have that $X_{e}$ is distributed as $\operatorname{Bin}[r, q]$.

The edge $e$ appears in $G_{\sigma}$ if and only if $e$ is part of a super-edge. Let $(i, j)$ be a fixed edge of $T$. The probability that $e$ crosses $\left(V_{\sigma, i}, V_{\sigma, j}\right)$ is $\frac{2}{t^{2}} \frac{n}{n-1}$. To see this, expose the position of $v$ under $\sigma$. Then $v$ lies in $V_{\sigma, i}$ or $V_{\sigma, j}$ with probability $2 \cdot \frac{n / t}{n}$. Conditioning, on this, the probability that $w$ lands in the other set is $\frac{n / t}{n-1}$. There are $t-1$ edges of $T$, and the events corresponding to $e$ belonging to different super-edges are mutually disjoint. So we have

$$
q=\frac{2(t-1)}{t^{2}}\left(1+\frac{1}{n-1}\right)
$$

which implies that

$$
\mathbb{E}\left[X_{e}\right]=r q=\frac{60}{\epsilon^{2}} \log n\left(1+\frac{1}{n-1}\right)
$$


and by Theorem 2.1, the probability that $X_{e}$ differs from its mean by more than .5єrq is bounded above by

$$
\exp \left\{-\frac{(.5 \epsilon)^{2}}{3} r q\right\}=o\left(n^{-3}\right)
$$

Hence with probability at least $1-o\left(n^{-1}\right)$, every edge is contained in $(1 \pm \epsilon) \frac{60}{\epsilon^{2}} \log n$ of the $G_{i}$ 's.

Lemma 3.3. Suppose that $\epsilon^{6} n p^{4} \gg \log ^{3} n$. Then after Step $\boldsymbol{P} 3$ of Procedure 1, with probability $1-o(1)$, each $\widehat{G}_{i}$ is a $\left(7 \epsilon, \frac{p}{\kappa}\right)$-regular blow up of $T$.

Proof. Since $r=\Theta\left(\log n / \epsilon^{2}\right)=o(n)$, by our assumptions on $n, p$ and $\epsilon$, we may assume that at the beginning of step P3, each of $G_{1}, \ldots, G_{r}$ is a $(2 \epsilon, p)$-regular blow up of $T$ and each edge of $G$ appears in $(1 \pm \epsilon) \kappa$ of the $G_{i}$ 's.

Now consider a single $\widehat{G}_{i}$. We will show that with sufficiently high probability, this is a $\left(7 \epsilon, \frac{p}{\kappa}\right)$-regular blow up of $T$. This means we must show that across super-edges, all degrees and co-degrees are very typically correct. The super-edges of $\widehat{G}_{i}$ are the same as those in $G_{i}$, but the edge density is lower by a factor of approximately $\kappa$ since each edge of $G_{i}$ chose to be in $\widehat{G}_{i}$ with probability approximately $1 / \kappa$.

Let $v$ be an arbitrary vertex of $\widehat{G}_{i}$, and let $\widehat{N}_{v}$ represent the degree of $v$ across a superedge in $\widehat{G}_{i}$. If we let $Z_{v}$ represent the degree of $v$ across this super-edge in $G_{i}$, then since $G_{i}$ is a $(2 \epsilon, p)$-regular blow up of $T$, we have that $Z_{v} \geqslant(1-2 \epsilon) \nu p$. Each of these $Z_{v}$ edges is included in $\widehat{G}_{i}$ independently with probability $\frac{1}{(1 \pm \epsilon) \kappa}$. So $\widehat{N}_{v}$ stochastically dominates the distribution

$$
\operatorname{Bin}\left[Z_{v}, \frac{1}{(1+\epsilon) \kappa}\right] \text {. }
$$

Thus

$$
\mathbb{E}\left[\widehat{N}_{v}\right] \geqslant(1-4 \epsilon) \nu \frac{p}{\kappa}
$$

and Theorem 2.1 tells us that

$$
\widehat{N}_{v} \geqslant(1-6 \epsilon) \nu \frac{p}{\kappa}
$$

with probability $1-o\left(n^{-K}\right)$ for any positive constant $K$ as long as

$$
\epsilon^{2} n \frac{p}{\kappa} \gg \log n \Longleftrightarrow \epsilon^{4} n p \gg \log ^{2} n \text {. }
$$

Now for co-degrees, we let $v, w$ be vertices in $\widehat{G}_{i}$ and let $\widehat{N}_{v, w}$ represent the co-degree of $v$ and $w$ across a super-edge in $\widehat{G}_{i}$. We let $Z_{v, w}$ represent the co-degree of $v$ and $w$ across this super-edge in $G_{i}$. Applying $(2 \epsilon, p)$-regularity of $G_{i}$, we have that $Z_{v, w} \leqslant(1+2 \epsilon) \nu p^{2}$. Each of these vertices remains a common neighbor of $v$ and $w$ in $\widehat{G}_{i}$ with probability $\left(\frac{1}{(1 \pm \epsilon) \kappa}\right)^{2}$, so $\widehat{N}_{v, w}$ is stochastically dominated by

$$
\operatorname{Bin}\left[Z_{v, w},\left(\frac{1}{(1-\epsilon) \kappa}\right)^{2}\right] \text {. }
$$


Thus

and Theorem 2.1 gives that

$$
\mathbb{E}\left[\widehat{N}_{v, w}\right] \leqslant(1+5 \epsilon) \nu\left(\frac{p}{\kappa}\right)^{2}
$$

$$
\widehat{N}_{v, w} \leqslant(1+7 \epsilon) \nu\left(\frac{p}{\kappa}\right)^{2}
$$

with probability $1-o\left(n^{-K}\right)$ for any positive constant $K$ as long as

$$
\epsilon^{2} n\left(\frac{p}{\kappa}\right)^{2} \gg \log n \Longleftrightarrow \epsilon^{6} n p^{2} \gg \log ^{3} n
$$

Taking a union bound over choices of vertices, super-edges and $\widehat{G}_{i}$, we conclude that with probability $1-o(1)$ each $\widehat{G}_{i}$ is a $\left(7 \epsilon, \frac{p}{\kappa}\right)$-regular blow-up of $T$.

To prove Theorem 1, we will apply the following result of Frieze and Krivelevich, which shows that any $(\epsilon, p)$-regular pair can have almost all of its edges covered by edge-disjoint perfect matchings.

Lemma 3.4 (Frieze, Krivelevich [6]). Suppose $(A, B)$ is an $(\eta, d)$-regular pair with $|A|=$ $|B|=\nu$ and $\eta^{4 / 3} d^{2} \nu \gg 1$ for some small value $\eta \ll 1$. Then $(A, B)$ contains a collection of $\left(1-\eta^{1 / 3}\right) d \nu$ edge-disjoint perfect matchings.

Proof of Theorem 1. Let $G$ be an $(\epsilon, p)$-regular graph on $n$ vertices with $\epsilon^{6} n p^{4} \gg \log ^{3} n$. Apply Procedure 1. Our conditions on $\epsilon, p$, and $n$ allow us to apply Lemma 3.3 and to conclude that at the end of Step $\mathbf{P} 3$ of Procedure 1, every $\widehat{G}_{i}$ is a $\left(7 \epsilon, \frac{p}{\kappa}\right)$-regular blow up of $T$. We also have that each edge of $G$ appears in exactly one of the $\widehat{G}_{i}$.

Consider one of the $\widehat{G}_{i}$, and call its $t-1$ super-edges $Q_{1}, \ldots, Q_{t-1}$. On each of these super-edges, we apply Lemma 3.4 with $\nu=n / t, \eta=7 \epsilon$ and $d=\frac{p}{\kappa}$. Then we have that each $Q_{j}$ contains a collection of edge-disjoint perfect matchings $\mathcal{M}_{j}$ of size at least

$$
s:=\left(1-(7 \epsilon)^{1 / 3}\right) \frac{p}{\kappa} \frac{n}{t} .
$$

Now select arbitrary matchings $M_{1} \in \mathcal{M}_{1}, M_{2} \in \mathcal{M}_{2}, \ldots, M_{t-1} \in \mathcal{M}_{t-1}$. Observe that

$$
M_{1} \cup M_{2} \cup \cdots \cup M_{t-1}
$$

is a $T$ factor since the super-edge structure of $\widehat{G}_{i}$ is isomorphic to $T$. We may thus extract at least $s$ edge-disjoint $T$-factors from $\widehat{G}_{i}$. Indeed, we may do this for each of $\widehat{G}_{1}, \ldots, \widehat{G}_{r}$. Tree factors extracted from distinct $\widehat{G}_{i}$ are edge-disjoint.

In total, the number of edges covered by these tree factors is at least

$$
s \cdot \frac{n}{t} \cdot(t-1) \cdot r=\left(1-(7 \epsilon)^{1 / 3}\right) \frac{n^{2}}{2} p,
$$

while the $(\epsilon, p)$-regularity of $G$ tells us that $G$ had at most $(1+\epsilon) \frac{n^{2}}{2} p$ edges total. So, the total fraction covered is at least

$$
\frac{1-(7 \epsilon)^{1 / 3}}{1+\epsilon} \geqslant 1-2 \epsilon^{1 / 3}
$$

as long as $\epsilon$ is sufficiently small. 


\section{Proof of Theorem 2}

Direct application of Theorem 1 for pseudo-random graphs gives a packing result for random graphs with $p \gg n^{-1 / 4} \log ^{3 / 4} n$. In this section, we use additional properties of random graphs to improve the range of $p$ to $p \gg \frac{\log ^{2} n}{n}$. For this, we will apply the following result, which is an analogue of Lemma 3.4 for the fully random graph setting.

Lemma 4.1 (Frieze, Krivelevich [6]). Let $G$ be a random bipartite graph with sides $A, B$ of size $|A|=|B|=\nu$, where each edge appears independently with probability at least $p=p(\nu)$. Assume that $p(\nu) \gg \frac{\log \nu}{\nu}$. Then with probability $1-o\left(\nu^{-1}\right), G$ contains a family of $(1-\delta) \nu p$ edge-disjoint perfect matchings, where

$$
\delta=\left(\frac{16 \log \nu}{\nu p}\right)^{1 / 2} .
$$

Proof of Theorem 2. The proof of this theorem is essentially identical to that in the previous section. Run Procedure 1 on a random graph $G=G_{n, p}$ with $n$ a multiple of $t$, and let $\nu=n / t$. Since $G$ is random, after Step $\mathbf{P} 1$, each super-edge of each $G_{i}$ is a copy of $B_{\nu, \nu, p}$, the random bipartite graph with parts of size $\nu$ and edge probability $p$. The proof of Lemma 3.2 applied to $G_{n, p}$ instead of an $(\epsilon, p)$-regular graph gives us that with probability $1-o\left(n^{-1}\right)$, each edge appears in $(1 \pm \epsilon) \kappa$ of the $G_{i}$ 's where $\kappa=\frac{60}{\epsilon^{2}} \log n$.

Conditioning on this, we see that after Step P3 of Procedure 1, in a particular $\widehat{G}_{i}$, across a super-edge $(A, B)$, each pair $(a, b), a \in A, b \in B$ is an edge of $\widehat{G}_{i}$ with probability at least

$$
q:=p \cdot \frac{1}{(1+\epsilon) \kappa} \gg \frac{\log n}{n}=\Theta\left(\frac{\log \nu}{\nu}\right) .
$$

So, we may apply Lemma 4.1 to each of the $(t-1) r$ super-edges in the $\widehat{G}_{i}$ 's. Since $(t-1) r \ll \nu$, we have that whp, each of the $(t-1) r$ super-edges satisfies the conclusion of the lemma.

Consider one of the $\widehat{G}_{i}$ 's and suppose that we call its $t-1$ super-edges $Q_{1}, \ldots, Q_{t-1}$. Then we have that each $Q_{j}$ contains a collection of edge-disjoint perfect matchings $\mathcal{M}_{j}$ of size at least

$$
s:=(1-\delta) q \nu
$$

Note that $\delta$ is bounded by

$$
\left(\frac{16 \log \nu}{\nu \frac{p}{(1+\epsilon) \kappa}}\right)^{1 / 2}=O(\epsilon) .
$$

As before, selecting arbitrary matchings $M_{1} \in \mathcal{M}_{1}, M_{2} \in \mathcal{M}_{2}, \ldots, M_{t-1} \in \mathcal{M}_{t-1}$ gives a $T$-factor

$$
M_{1} \cup M_{2} \cup \cdots \cup M_{t-1} .
$$

We may thus extract at least $s$ edge-disjoint $T$-factors from $\widehat{G}_{i}$ and do this for each of $\widehat{G}_{1}, \ldots, \widehat{G}_{r}$. In total, the number of edges covered will be at least

$$
s \cdot \nu \cdot(t-1) \cdot r=(1-O(\epsilon)) \frac{n^{2}}{2} p
$$


which is the desired number of edges since the graph has at most $(1+\epsilon)\left(\begin{array}{l}n \\ 2\end{array}\right) p$ edges total whp by Theorem 2.1 .

\section{$5 \quad$ Proof of Theorem 3}

We conclude by introducing a different argument which "bootstraps" Theorem 1 to drive the range of $p$ all the way down to about $\frac{\log n}{n}$. Note that this is essentially the limit, because for $p$ below $\frac{\log n}{n}$, the random graph typically contains isolated vertices, and therefore finding even a single $T$-factor would be impossible.

Proof of Theorem 3. Let $\tau_{1}$ be the smallest value of $\tau$ for which Theorem 1 applies for $\left(\epsilon^{3}, 1\right)$-regular graphs on $\tau$ vertices. Let $\tau_{0}=\max \left\{\tau_{1}, \epsilon^{-3}\right\}$, and assume that $\tau \geqslant \tau_{0}$, with $t \mid \tau$. Define $C=\tau \epsilon^{-2}$. The idea of the proof is to first split the vertex set of $G \sim G_{n, p}$ into $\tau$ parts $V_{1} \cup \cdots \cup V_{\tau}$ of size $\ell$ each. We then think of these parts as vertices of the complete graph on $\tau$ vertices and note that the complete graph $K_{\tau}$ is a $(\delta, 1)$-regular graph for any $\delta \geqslant 1 / \tau$. We apply Theorem 1 to find a collection of edge-disjoint $T$-factors which cover almost all the edges of $K_{\tau}$. Each edge appearing in a $T$-factor of $K_{\tau}$ corresponds to a random bipartite graph $B_{\ell, \ell, p}$ in $G$. To each such bipartite graph we apply Lemma 4.1. We must show that the total number of edges covered is at least $(1-O(\epsilon))\left(\begin{array}{l}n \\ 2\end{array}\right) p$ since by Theorem 2.1, $G_{n, p}$ has at most $(1+\epsilon)\left(\begin{array}{l}n \\ 2\end{array}\right) p$ edges total whp.

We now analyze this procedure quantitatively. We fail to cover edges in three ways: when they are within a single $V_{i}$, when they are between a pair $\left(V_{i}, V_{j}\right)$ which is not covered by a $T$-factor, and when they are within a $T$-factor edge, but missed by Lemma 4.1. We must ensure that the total fraction missed is $O(\epsilon)$. To this end, note that the first omission loses only at most $\frac{1}{\tau}$-fraction of the edges, while the second loses at most $2 \delta^{1 / 3}$-fraction of the edges by Theorem 1. Therefore, as long as $\tau>\tau_{0}=\epsilon^{-3}$ (which implies that $K_{\tau}$ is $(\delta, 1)$-regular with $\delta=\epsilon^{3}$ ), the total loss from the first two types is only $O(\epsilon)$. Note that this bounded loss is completely deterministic.

We control the third type of omission using the randomness in $G_{n, p}$. The bipartite graph between every pair $\left(V_{i}, V_{j}\right)$ covered by a $T$-factor in $K_{\tau}$ is a copy of the random bipartite graph $B_{\ell, \ell, p}$. If we apply Lemma 4.1 to such a graph, we obtain a collection $\mathcal{M}_{i, j}$ of at least $\left(1-\left(\frac{16 \log \ell}{\ell p}\right)^{1 / 2}\right) \ell p$ edge-disjoint matchings with probability $1-o\left(\ell^{-1}\right)$. Since we are proving that $G_{n, p}$ satisfies $\mathcal{P}(\epsilon) \mathbf{w h p}, \tau$ is a constant while $n \rightarrow \infty$, and therefore $\tau^{2} \ll \ell$; a union bound then implies that whp, every pair $\left(V_{i}, V_{j}\right)$ from the $T$-factor of $K_{\tau}$ contains such a collection $\mathcal{M}_{i, j}$. As in the proofs of our other two results, these perfect matchings combine to form $T$-factors of the full $n$-vertex graph. It therefore remains only to show that the fractional loss can be kept below $O(\epsilon)$. For this, we use $p>\frac{C \log n}{n}$, and simplify:

$$
\left(\frac{16 \log \ell}{\ell p}\right)^{1 / 2}<\left(\frac{16 \log \ell}{\ell \frac{C \log (\tau \ell)}{\tau \ell}}\right)^{1 / 2}=O\left(\left(\frac{\tau}{C}\right)^{1 / 2}\right)=O(\epsilon)
$$

since $C=\tau \epsilon^{-2}$. 


\section{References}

[1] Deepak Bal and Alan Frieze. Packing tight Hamilton cycles in uniform hypergraphs. SIAM J. Discrete Math., 26(2):435-451, 2012.

[2] Béla Bollobás and Alan M. Frieze. On matchings and Hamiltonian cycles in random graphs. In Random graphs '83 (Poznań, 1983), volume 118 of North-Holland Math. Stud., pages 23-46. North-Holland, Amsterdam, 1985.

[3] P. Erdős and A. Rényi. On the existence of a factor of degree one of a connected random graph. Acta Math. Acad. Sci. Hungar., 17:359-368, 1966.

[4] Alan Frieze and Michael Krivelevich. On packing Hamilton cycles in $\epsilon$-regular graphs. J. Combin. Theory Ser. B, 94(1):159-172, 2005.

[5] Alan Frieze and Michael Krivelevich. On two Hamilton cycle problems in random graphs. Israel J. Math., 166:221-234, 2008.

[6] Alan Frieze and Michael Krivelevich. Packing Hamilton cycles in random and pseudorandom hypergraphs. Random Structures and Algorithms, 41(1):1-22, 2012.

[7] Alan Frieze, Michael Krivelevich, and Po-Shen Loh. Packing tight Hamilton cycles in 3-uniform hypergraphs. Random Structures and Algorithms, 40(3):269-300, 2012.

[8] Alan Frieze and Boris Pittel. Perfect matchings in random graphs with prescribed minimal degree. In Mathematics and Computer Science. III, Trends Math., pages 95-132. Birkhäuser, Basel, 2004.

[9] Svante Janson, Tomasz Łuczak, and Andrzej Rucinski. Random graphs. WileyInterscience Series in Discrete Mathematics and Optimization. Wiley-Interscience, New York, 2000.

[10] Fiachra Knox, Daniela Kühn, and Deryk Osthus. Approximate Hamilton decompositions of random graphs. Random Structures and Algorithms, 40(2):133-149, 2012.

[11] Fiachra Knox, Daniela Kühn, and Deryk Osthus. Edge-disjoint Hamilton cycles in random graphs, Random Structures and Algorithms, to appear. doi:10.1002/rsa.20510

[12] Michael Krivelevich and Wojciech Samotij. Optimal packings of Hamilton cycles in sparse random graphs. SIAM J. Discrete Math., 26(3):964-982, 2012.

[13] Daniela Kühn and Deryk Osthus. Hamilton decompositions of regular expanders: Applications, J. Combin. Theory Ser. B, 104:1-27, 2014.

[14] Justyna Kurkowiak. On disjoint perfect tree-matchings in random graphs. Australas. J. Combin., 19:47-54, 1999.

[15] Tomasz Łuczak and Andrzej Ruciński. Tree-matchings in graph processes. SIAM J. Discrete Math., 4(1):107-120, 1991.

[16] Colin McDiarmid. Concentration. In Probabilistic Methods for Algorithmic Discrete Mathematics, volume 16 of Algorithms Combin., pages 195-248. Springer, Berlin, 1998.

[17] E. Shamir and E. Upfal. On factors in random graphs. Israel J. Math., 39(4):296-302, 1981. 